\title{
A Consensus Definition of Supratotal Resection for Anatomically Distinct Primary Glioblastoma: An AANS/CNS Section on Tumors Survey of Neurosurgical Oncologists
}

\author{
Maureen Rakovec \\ Johns Hopkins University School of Medicine \\ Adham M. Khalafallah \\ University of Miami Miller School of Medicine: University of Miami School of Medicine \\ Oren Wei \\ Johns Hopkins University \\ David Day \\ Johns Hopkins University \\ Jason P. Sheehan \\ University of Virginia Health System \\ Jonathan H. Sherman \\ West Virginia University \\ Debraj Mukherjee ( $\nabla$ dmukher1@jhmi.edu ) \\ Johns Hopkins University School of Medicine https://orcid.org/0000-0002-5403-8237
}

\section{Research Article}

Keywords: glioblastoma, neuro-oncology, supratotal resection, crowdsourcing

Posted Date: November 30th, 2021

DOI: https://doi.org/10.21203/rs.3.rs-1098449/v1

License: (c) (i) This work is licensed under a Creative Commons Attribution 4.0 International License.

Read Full License 


\section{Abstract}

\section{Introduction:}

Supratotal resection (SpTR) of glioblastoma may be associated with improved survival, but published results have varied in part from lack of consensus on the definition and appropriate use of SpTR. A previous small survey of neurosurgical oncologists with expertise performing SpTR found resection 1-2 $\mathrm{cm}$ beyond contrast enhancement was an acceptable definition and glioblastoma involving the right frontal and bilateral anterior temporal lobes were considered most amenable to SpTR. The general neurosurgical oncology community has not yet confirmed the practicality of this definition.

\section{Methods}

Seventy-six general neurosurgical oncology members of the AANS/CNS Tumor Section were surveyed using a crowdsourcing approach. Participants were presented with 11 definitions of SpTR and rated each definition's appropriateness. Participants additionally reviewed magnetic resonance imaging for 10 anatomically distinct glioblastomas and assessed the tumor location's eloquence, perceived equipoise of enrolling patients in a randomized trial comparing gross total to SpTR, and their personal treatment plans.

\section{Results}

Fifty-two neurosurgeons (73.2\%) agreed that resection $1-2 \mathrm{~cm}$ beyond contrast enhancement was an acceptable definition for SpTR. Cases were divided into three anatomically distinct groups by perceived equipoise between gross total and SpTR. The best clinical trial candidates were right anterior temporal $(n=58,76.3 \%)$ and right frontal $(n=55,73.3 \%)$ glioblastomas.

\section{Conclusion}

Support exists within the neurosurgical oncology community to adopt the proposed consensus definition of SpTR of glioblastoma and to treat right anterior temporal and right frontal glioblastomas using SpTR. A smaller proportion of general neurosurgical oncologists than SpTR experts consider SpTR feasible in the left anterior temporal lobe.

\section{Introduction}

Glioblastoma (GBM) is the most common adult primary central nervous system cancer, accounting for $45.6 \%$ of all primary brain malignancies with an annual incidence of 3.1 per $100,000[1,2]$. It is highly aggressive with histopathological characteristics including necrosis, endothelial proliferation, anaplasia, and high mitotic rates [3]. GBM is also resistant to current treatment protocols. The median survival of 
GBM patients is only approximately 15 months following the standard of care: surgery, radiotherapy, and temozolomide $[1,4]$.

GBM's tendency to infiltrate the parenchyma beyond contrast-enhancing regions seen on magnetic resonance imaging (MRI) contributes to its aggressiveness [1,5]. While gross total resection (GTR) of gadolinium-enhancing tumor is correlated with increased survival time and decreased disease progression compared to subtotal resection (STR) or biopsy, recurrence is usually inevitable at or near the primary resection site [6-10]. Encouraged by studies that established a relationship between supratotal resection (SpTR) of low grade gliomas and improved overall survival (OS) without increased incidence of new, post-operative deficits, neurosurgical oncologists have begun to use this technique with IDH-wildtype GBM $[11,12]$.

Recent systematic reviews have demonstrated that SpTR of GBM can improve OS, likely by excising noncontrast enhancing neoplastic cells in high-signal, T2 fluid-attenuated inversion recovery (FLAIR) regions on MRI [11, 13-15]. However, authors observed heterogeneity in the magnitude of OS improvement achieved using SpTR, attributable to differences in how SpTR was defined. Further, a delicate balance between tumor burden reduction and preservation of neurologic function must be achieved when SpTR is used. This has encouraged its use in relatively non-eloquent areas of the brain, though the effect of tumor location on OS after SpTR has not yet been definitively established.

Our group has previously used crowdsourcing, a technique that draws on the collective knowledge of a community, to address the need for a single definition for SpTR in GBM, as well as to produce consensus on the anatomical locations most amenable to this technique [16]. Based on the judgment of 21 members of the neurosurgical oncology community with expertise performing SpTR to treat GBM, we previously proposed GTR plus resection 1 to $2 \mathrm{~cm}$ beyond contrast-enhancement as an appropriate consensus definition of SpTR, with GBMs in the right frontal, right anterior temporal, and left anterior temporal lobes being the best SpTR candidates. This study again uses crowdsourcing while investigating potential support for a SpTR strategy in GBM among a larger, more general neurosurgical oncology community.

\section{Methods}

\section{Survey Creation}

A comprehensive literature search identified eleven definitions for SpTR of GBM and four definitions of eloquence (Supplement) [14, 17-31]. To investigate the anatomic locations where SpTR was considered an appropriate treatment for GBM, preoperative MRI data was sourced from 10 de-identified adult $(\geq 18$ years) GBM patients (Supplement). This study was exempt from obtaining patient consent; all radiographic data was de-identified as required by Health Insurance Portability and Accountability Act (HIPAA) regulations and institutional review board protocol (IRB00196609).

\section{Survey Distribution and Data Collection}


The survey and its responses were created, distributed, and stored using Qualtrics software, Version XM (Qualtrics, Provo, UT). An invitation to participate in a 72-question online survey (eAppendix) was sent via email to all members of the AANS/CNS Section on Tumors in two waves between January 20 and February 25, 2021. Demographic information was collected from each participant (Supplement). To reduce survey fatigue, participants were informed that they could complete the survey in multiple sittings.

For each of the eleven definitions of SpTR, survey respondents were asked if they strongly agreed, agreed, disagreed, or strongly disagreed that it was appropriate to use in a potential future clinical trial assessing SpTR for GBM. They then identified which of four definitions of eloquence was consistent with how they described areas of the brain; if no option was appropriate, they provided their own definition under the option "other."

For each of the ten anatomically distinct GBM cases, survey respondents reviewed MR images and categorized the eloquence of the tumor's location, indicated whether it would be reasonable to randomize it to SpTR or GTR, and identified their personal treatment plan and any adjunct perioperative techniques they would use (Supplement). Consensus on each case's eloquence, equipoise, or treatment plan was defined as at least $70 \%$ agreement among the respondents, following past conventions [32, 33].

\section{Statistical Analysis}

Statistical analyses were performed with $R$ version 4.0.3 and Microsoft Excel 2021. $P$ values $<0.05$ were considered statistically significant (Supplement). A "resectability index" (RI) was calculated for each case to quantify and compare the perceived level of resectability of each GBM [34] (Supplement).

\section{Results}

\section{Demographic Characteristics of Survey Respondents}

A total of 76 neurosurgical oncologists completed the survey, representing $34.0 \%$ of the 223 members of the AANS/CNS Tumor Section invited to participate who opened the survey. The cohort's median time practicing neurosurgery was 10 years (interquartile range, IQR 5-25 years, Table 1). Most respondents $(n=61,80.3 \%)$ practiced in an academic setting; ten (13.2\%) were employed at a community hospital, and $11(17.7 \%)$ worked in private practice. The scope of practice of most neurosurgeons surveyed included intra-axial tumors ( $n=60,78.9 \%$ ). A total of 32 participants $(42.1 \%)$ formally completed a neuro-oncology fellowship. The median number of SpTRs for GBM performed by the cohort annually and in total were 2 (IQR 0.8-5) and 20 (IQR 5-50), respectively. 
Table 1

Practice characteristics of neurosurgeons who participated in this crowdsourcing survey.

\begin{tabular}{|c|c|}
\hline Variable & All respondents $(n=76)$ \\
\hline \multicolumn{2}{|l|}{ Years in Practice* } \\
\hline Mean (SD) & $15.4(12.8)$ \\
\hline Median (IQR) & $10(5-25)$ \\
\hline \multicolumn{2}{|l|}{ Practice Setting, n (\%) } \\
\hline Academic hospital & $61(80.3)$ \\
\hline Community hospital & $10(13.2)$ \\
\hline Academic-affiliated private practice & $7(9.2)$ \\
\hline Private practice with partners & $4(8.5)$ \\
\hline \multicolumn{2}{|l|}{ Practice Focus, n (\%) } \\
\hline Intra-axial tumors & $60(78.9)$ \\
\hline Skull base tumors & $40(52.6)$ \\
\hline General neurosurgery & $33(43.4)$ \\
\hline Spine tumors & $19(25.0)$ \\
\hline \multicolumn{2}{|l|}{ Neuro-oncology Fellowship, n (\%) } \\
\hline Yes & $32(42.1)$ \\
\hline No & $44(57.9)$ \\
\hline \multicolumn{2}{|l|}{ Intraoperative Techniques Utilized, n (\%) } \\
\hline Functional MRI & $59(80.8)$ \\
\hline Diffusion Tensor Imaging & $56(76.7)$ \\
\hline Awake craniotomy with speech mapping & $53(72.6)$ \\
\hline Asleep motor mapping & $55(75.3)$ \\
\hline Awake craniotomy with motor mapping & $51(69.9)$ \\
\hline 5-ALA fluorescence guided surgery & $38(52.1)$ \\
\hline Ultrasound & $44(60.3)$ \\
\hline MRI & $23(31.5)$ \\
\hline
\end{tabular}

*Missing 5 for years in practice, 7 for annual and total SpTR counts 


\begin{tabular}{|lc|}
\hline Variable & All respondents $(\mathbf{n}=\mathbf{7 6})$ \\
\hline Intraoperative Mapping Training, $\mathbf{n}(\%)$ & \\
\hline Yes & $37(48.7)$ \\
\hline No & $39(51.3)$ \\
\hline SpTR of GBM Performed, Median (IQR)* & \\
\hline Annually & $2(0.8-5)$ \\
\hline Total & $20(5-50)$ \\
\hline *Missing 5 for years in practice, 7 for annual and total SpTR counts \\
\hline
\end{tabular}


Table 2

A. Agreement and disagreement among 76 neurosurgical oncologists regarding 11 published definitions of SpTR for GBM. 2B. The neurosurgical oncologists were also provided with four published definitions of eloquence and asked to indicate how they defined eloquent brain structures.
2A. Definitions of SpTR*
Disagree or Strongly
Disagree, $\mathrm{n}(\%)$

Agree or

Strongly Agree, $\mathrm{n}$

(\%)

\section{Extent of T2 FLAIR Resection}

Any decrease in post-op FLAIR volume

$32(45.1)$

39 (54.9)

GTR $+>25 \%$ of FLAIR abnormality region

$37(52.1)$

34 (47.9)

GTR $+>45-50 \%$ of FLAIR abnormality region

$43(62.3)$

26 (37.7)

GTR $+>54 \%$ of FLAIR abnormality region

$44(62.9)$

$26(37.1)$

GTR $+>75 \%$ of FLAIR abnormality region

39 (55.7)

31 (44.3)

GTR $+100 \%$ of FLAIR abnormality region

27 (37.5)

$45(62.5)$

\section{Other Extent of Resection Definitions}

Any resection beyond GTR

$21(29.2)$

$51(70.8)$

GTR + resection of edematous tissue involved radiographically normal gyrus

GTR + any resection of non-contrast enhanced disease

14 (19.7)

$57(80.3)$

Resection 1-2 $\mathrm{cm}$ beyond contrast enhancement

$19(26.8)$

52 (73.2)

GTR + resection of surrounding non-eloquent, radiographically normal cortex and white matter

2B. Definitions of Eloquence*

Agree, n (\%)

\section{Reference areas of the brain that speak to readily identifiable neurologic function and, if injured, result in a disabling neurologic deficit.}

$50(67.6)$

Areas of the brain are eloquent if they are functional as determined by intraoperative functional mapping.

Areas of the brain are eloquent if they are functional as determined by preoperative functional MRI.

All areas of the brain are eloquent.

Other**

$2(2.7)$

*Missing 1 for SpTR definitions, Missing 3 for eloquence definitions **"Other" includes "Combination of 1 and $3-$ cortex defined by functional mapping, subcortical structures defined by known anatomical reference" and "1,2, and 3"

\section{Definitions of SpTR and Eloquence}


Most neurosurgeons surveyed $(n=57,80.3 \%)$ agree or strongly agree that GTR plus resection of any noncontrast enhanced disease is an appropriate definition for SpTR (Table 2A). Compared to the cohort of expert neurosurgical oncologists who previously provided their opinions on this definition, a two proportion z-test demonstrated that a similar fraction agreed or strongly agreed that it was fitting (Experts: $\mathrm{n}=18,85.7 \%$; Tumor Section: $\mathrm{n}=57,80.3 \%$; $\mathrm{p}=0.575$ ) [33]. GTR plus resection $1-2 \mathrm{~cm}$ beyond contrast-enhancing disease was the second most commonly endorsed definition, with 52 (73.2\%) neurosurgeons agreeing or strongly agreeing with this definition of SpTR. There were no significant relationships between demographic variables, including time in practice, practice setting, or SpTRs performed in the last year or in total, and support for this definition. When asked to indicate how they defined eloquence, over two-thirds of the cohort chose "reference areas of the brain that speak to readily identifiable neurologic function and, if injured, result in a disabling neurologic deficit" ( $n=50,67.6 \%$, Table 2B). Only five (6.8\%) neurosurgeons surveyed considered the entire brain eloquent.

\section{Agreement Among Surgeons on Eloquence of GBM Location and Treatment Plan}

There was consensus that GBMs in the left thalamic (97.3\%) and right occipital (70.7\%) regions were eloquent (Figure 1). Right anterior temporal (62.7\%) and right frontal (58.7\%) GBMs were considered located in non-eloquent regions by the majority of the cohort. There was no significant difference between the fractions of the cohort that described the right anterior temporal and right frontal GBMs in noneloquent locations in a two proportion z-test $(p=0.174)$. Neurosurgeons who considered the entire brain eloquent were more likely to label the right cerebellar GBM as near-eloquent $(p=0.0235)$ relative to those who define eloquence as "distinct areas of the brain with readily identifiable neurologic function"; there were no other correlations between surgeons' definition of eloquence and the perceived eloquence of the cases.

Figure 2 illustrates the treatment plans for each case and corresponding RI and variance calculations. The majority of the cohort agreed that right anterior temporal and right frontal GBMs were amenable to SpTR (69.3\% and 58.7\%, respectively). A smaller percentage (40.0\%) chose SpTR as their treatment plan for the left anterior temporal GBM; neurosurgeons who described this location as eloquent or neareloquent were significantly less likely in chi-square analysis to recommend SpTR as the treatment plan $(p=0.039)$. However, the cohort still agreed that an aggressive treatment plan such as SpTR or GTR should be pursued for this GBM, as demonstrated by its relatively high RI value (4.37). A significant number of those who would resect these three GBMs indicated that they would utilize 5-Aminolevulinic acid (5-ALA) fluorescence as an intra-operative adjunct (right anterior temporal: $n=42$; right frontal: $n=48$; left anterior temporal: $n=50$; Supplement). The cohort was most divided on the optimal treatment plan for the right insular GBM; this case had the lowest sum of squares and therefore the greatest variance between proposed treatment plans (684).

\section{Agreement Among Surgeons Regarding Equipoise Between SpTR and GTR}


When asked which cases would be amenable to enrollment in a clinical trial that would randomize patients to either SpTR or GTR, the neurosurgical oncologists reached consensus that right anterior temporal $(n=58,76.3 \%)$ and right frontal $(n=55,73.3 \%)$ GBMs would probably or definitely be appropriate (Table 3). Though not reaching the $70 \%$ threshold for consensus, a significant percentage of the cohort $(n=48,64.0 \%)$ also considered the left anterior temporal GBM to be a randomization candidate.

Consensus within the cohort was greatest to not randomize patients with a left thalamic GBM to such a clinical trial $(n=63,84.0 \%)$ as indicated by its high sum of squares $(2168.0)$. 
Table 3

A crowdsourcing approach revealed consensus among neurosurgeons regarding the equipoise of GTR or SpTR for anatomically distinct GBMs. A higher total sum of squares indicates greater agreement between surgeons to randomize patients to SpTR or GTR. Group A: >70\% strong consensus to randomize, Group B:

no overwhelming consensus, Group C: $>70 \%$ strong consensus to not randomize.

\begin{tabular}{|c|c|c|c|c|c|}
\hline $\begin{array}{l}\text { Case } \\
\#\end{array}$ & $\begin{array}{l}\text { Tumor } \\
\text { Location }\end{array}$ & $\begin{array}{l}\text { Probably or } \\
\text { Definitely No, } \\
\text { N (\%) }\end{array}$ & $\begin{array}{l}\text { Maybe, } \\
\mathrm{N}(\%)\end{array}$ & $\begin{array}{l}\text { Probably or Definitely } \\
\text { Yes, N (\%) }\end{array}$ & $\begin{array}{l}\text { Total Sum of } \\
\text { Squares }\end{array}$ \\
\hline \multicolumn{6}{|l|}{ Group A } \\
\hline 2 & $\begin{array}{l}\text { R Ant } \\
\text { Temporal }\end{array}$ & $13(17.1)$ & $5(6.6)$ & 58 (76.3) & 1632.7 \\
\hline 4 & R Frontal & $7(9.3)$ & $\begin{array}{l}13 \\
(17.3)\end{array}$ & 55 (73.3) & 1368.0 \\
\hline \multicolumn{6}{|l|}{ Group B } \\
\hline 9 & $\begin{array}{l}\text { L Ant } \\
\text { Temporal }\end{array}$ & 14 (18.7) & $\begin{array}{l}13 \\
(17.3)\end{array}$ & 48 (64.0) & 794.0 \\
\hline 8 & R Occipital & $28(37.3)$ & $\begin{array}{l}14 \\
(18.7)\end{array}$ & $33(44.0)$ & 194.0 \\
\hline 7 & R Parietal & $28(37.3)$ & $\begin{array}{l}13 \\
(17.3)\end{array}$ & $34(45.3)$ & 234.0 \\
\hline 1 & $\begin{array}{l}\mathrm{R} \\
\text { Cerebellum }\end{array}$ & $35(46.1)$ & $\begin{array}{l}10 \\
(13.2)\end{array}$ & $31(40.8)$ & 360.7 \\
\hline 5 & $\begin{array}{l}\text { R Post } \\
\text { Temporal }\end{array}$ & $30(40.0)$ & $\begin{array}{l}18 \\
(24.0)\end{array}$ & $27(36.0)$ & 78.0 \\
\hline 6 & R Insular & $36(48.0)$ & $\begin{array}{l}15 \\
(20.0)\end{array}$ & $24(32.0)$ & 222.0 \\
\hline 3 & L Frontal & 39 (51.3) & $\begin{array}{l}13 \\
(17.1)\end{array}$ & $24(31.6)$ & 340.7 \\
\hline
\end{tabular}

\section{Group C}

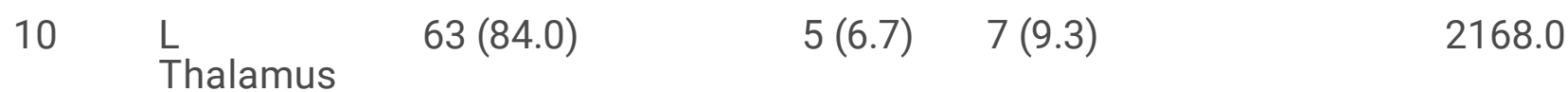

R: Right; L: Left; Ant: Anterior; Post: Posterior

*Missing 1 for cases 4-10

Variations in the Applicability of SpTR by GBM Location Between General Neurosurgical Oncologists and Experienced SpTR Providers

Previously, 21 neurosurgical oncologists with experience performing SpTR for GBM provided their opinions on the appropriateness of SpTR as a treatment plan for the 10 cases included in this survey [16]. 
Compared to the experts, a significantly smaller fraction of AANS/CNS Tumor Section members recommended SpTR as their treatment plan of choice for GBMs in two locations (Supplement). For the right frontal GBM, 18 (85.7\%) SpTR experts chose SpTR as their treatment option, while a significantly smaller fraction of the Tumor Section cohort $(n=44,58.7 \%)$ recommended SpTR $(p=0.023)$. Notably, a similar fraction of experts and generalists recommended SpTR for the right anterior temporal GBM (Experts: $n=14,70 \%$; Tumor Section: $n=52,69.3 \% ; p=0.999)$. SpTR was chosen as the preferred treatment for the left anterior temporal GBM by $15(78.9 \%)$ neurosurgeons in the expert cohort and $30(40.0 \%)$ in the Tumor Section cohort $(p=0.004)$.

\section{Discussion}

This survey demonstrates crowdsourcing's ability to provide insights into complex neurosurgical debates. The neurosurgical oncologists who completed this survey varied significantly in their practice setting and focus, as well as their personal experience performing SpTR for GBM. However, consensus was still reached in this large, diverse group of neurosurgical oncologists on a number of questions about SpTR for GBMs in specific anatomical regions. This encourages further use of the "wisdom of the crowd" approach to inform decisions regarding patient care where no one treatment has been conclusively proven superior $[32,34]$.

These results also provide support for our previously proposed consensus definition of SpTR for GBM [16]. There is a considerable need to adopt a single definition, as the multitude of definitions currently in use has obscured the impact of SpTR on OS in GBM. The 2018 systematic review and meta-analysis by Jackson et al. including 11 studies and 810 patients reported a survival benefit with SpTR over GTR in 9 of 11 studies (SpTR median OS 12-54 months; GTR median OS 11-17.5 months) [14]. However, the authors noted significant heterogeneity between the methods used in the included studies, and secondarily elected to divide studies into three subgroups based on their definition of SpTR, including extent of T2 FLAIR resection, extended anatomical resection, and intra-operative 5-aminolevulinic acid (5ALA) fluorescence-guided resection. Meta-analysis in the more homogenous extended anatomical resection subgroup demonstrated a statistically significant 35\% lower risk of mortality among 88 GBM patients who underwent SpTR versus 95 patients who received GTR $(H R=0.65,95 \% \mathrm{Cl} 0.47-0.91 ; p=$ 0.003). This study, the largest systematic review to date on the efficacy of SpTR, supports the selection of a single, anatomically-based definition for SpTR.

Similar to our previous survey of 21 neurosurgical oncologists with experience performing SpTR for GBM, "GTR plus any resection of non-contrast enhanced disease" was the most commonly positively endorsed definition, with a similar fraction of each cohort agreeing or strongly agreeing that it was an acceptable definition for SpTR. However, as previously discussed, this definition would not standardize clinical research involving SpTR, provide a quantifiable benchmark for what can and cannot be classified as SpTR, or allow a neurosurgeon to determine when SpTR has been achieved using intraoperative navigation [16]. For these reasons and in light of the corpus of literature that demonstrates GBMs most often recur within $2 \mathrm{~cm}$ of the original contrast-enhancing tumor's margins, we recommend that "GTR 
plus resection 1-2 $\mathrm{cm}$ beyond contrast enhancement" be implemented as a standardized definition of SpTR for GBM in clinical practice and future research studies [15, 21, 35-40]. A consensus of the AANS/CNS Tumor Section members who completed this survey support the adoption of this definition.

Similar to the results of surveying neurosurgical oncologists with expertise performing SpTR for GBM, the AANS/CNS Tumor Section cohort considered right frontal and right anterior temporal GBMs to be located in non-eloquent regions, amenable to SpTR, and appropriate to enroll in a clinical trial comparing SpTR to GTR. Of note, while the Tumor Section cohort found the right frontal GBM appropriate to enroll in a SpTR vs. GTR clinical trial by consensus, it was mildly more reticent to choose SpTR as the treatment they would personally perform relative to the right anterior temporal GBM; while 52 (69.3\%) neurosurgeons thought SpTR was the best treatment for the right anterior temporal GBM, a smaller though still considerable fraction ( $n=44,58.7 \%$ ) would perform SpTR of the right frontal GBM. This trend does not appear to be associated with the relative perceived eloquence of the involved tissues but may be because the practice of performing lobectomy for GBM in the right temporal lobe is relatively well-known and supported by literature that demonstrates improved survival without increased frequency of postoperative deficits, while studies on the effect of SpTR specifically in the right frontal lobe are less common [41-43].

When previously surveyed, the $21 \mathrm{SpTR}$ experts also agreed by consensus that the left anterior temporal GBM was amenable to SpTR vs. GTR randomization. This current cohort of more general neurosurgical oncologists, however, did not reach the $70 \%$ threshold for consensus. Compared to those with SpTR expertise $(n=15,78.9 \%)$, a significantly smaller fraction of neurosurgical oncologists from the Tumor Section designated SpTR as their chosen treatment for this GBM $(n=30,40.0 \%, p=0.004)$. This relative reluctance of the general neurosurgical oncologists to perform SpTR in this area may be related to the perceived eloquence of surrounding tissue, which includes Broca's and Wernicke's areas, visual fibers, and auditory cortex. Inadvertently injuring adjacent tissue involved in language could have a severe impact on quality of life, but such deficits have also been associated with decreased survival [44, 45]. These risks can be reduced by performing an awake craniotomy with speech mapping for the resection of left anterior temporal GBMs $[12,46,47]$. Of the 72 respondents who selected which intraoperative adjunctive methods they would employ during the resection of a left anterior temporal GBM, $32(44.4 \%)$ indicated that they would use this technique; 53 of 76 survey respondents (72.6\%) reported that they routinely perform awake craniotomies with speech mapping in their practice. Increasing this modality's usage during the resection of left anterior temporal GBMs may encourage the wider neurosurgical community to consider performing SpTR for GBM in this region.

While this crowdsourcing survey is valuable for demonstrating consensus among the broader neurosurgical community on the preferred treatment of right frontal and anterior temporal GBMs, it is equally useful for revealing the anatomic locations of GBM where there is less agreement on optimal treatment. The proposed treatments for right insular GBM varied widely within the AANS/CNS Tumor Section cohort. While some felt that pursuing SpTR was reasonable, others recommended less aggressive interventions including LITT and SRS; even a simple majority could not agree on a treatment plan.

Page $12 / 19$ 
Historically, neurosurgeons have been hesitant to resect insular tumors due to this cortex's eloquence, complex shape, and relationship to the internal and middle cerebral arteries [48]. However, as the relationship between extent of resection and OS in insular high-grade gliomas has become clearer, increasing extent of resection while reducing morbidity using improved microsurgical and intraoperative adjunctive techniques has become increasingly recommended $[6,7,48,49]$. Alimohamadi et al., for instance, demonstrated the potential of adjuncts including fMRI, diffusion tensor imaging (DTI), and awake craniotomy with intraoperative speech and motor mapping to yield high extent of resection without increased mortality or new major neurologic morbidities [50]. Hervey-Jumper and Berger reinforce that awake craniotomy with cortical and subcortical stimulation mapping during insular glioma resection results in lower rates of neurological deficit than resections performed without mapping (with: 4.3\%; without: 19\%; $p=0.008$ ) [48]. Again, further study and more widespread adoption of these techniques may encourage more aggressive intervention for insular GBMs and longer survival times for these patients.

\section{Limitations}

The individuals who completed this survey were recruited via email invitation and were not financially compensated. If these neurosurgeons chose to participate because they hold strong opinions about SpTR, the internal validity of the results may have been influenced by response bias.

Participants were asked to make decisions about the treatment of GBM patients using only anatomical information. However, when making decisions about surgical treatment of GBM, clinical factors are necessarily taken into account. The cases included in this study were taken from non-elderly and functional patients, which may limit the applicability of the results to older or less robust populations. In addition, extent of resection is often dependent intra-operatively on data from 5-ALA fluorescence or other adjuncts. This information was not provided on the survey in order to directly investigate the relationship between proposed treatment plans and anatomy alone; however, this constraint also limited the results' generalizability.

\section{Conclusion}

Support exists among the general neurosurgical oncology community to adopt resection 1-2 $\mathrm{cm}$ beyond contrast enhancement as a standardized definition for future clinical work and research studies investigating SpTR for GBM. GBMs involving the right frontal and right anterior temporal lobes are considered most amenable to SpTR by a group of general neurosurgical oncologists. A discrepancy exists between the proportion of SpTR experts and general neurosurgical oncologists who consider SpTR feasible in the left anterior temporal lobe. These results support planning prospective studies, including future clinical trials, to reinforce the clinical utility of SpTR for GBMs in the right frontal and right anterior temporal lobes, and encourages further research and discussion about the applicability of SpTR to GBMs in the left anterior temporal lobe.

\section{Declarations}


Reporting Guidelines: We found no applicable reporting guidelines that would apply to this article. By following the EQUATOR reporting guidelines decision tree, (http://www.equatornetwork.org/wpcontent/uploads/2013/11/20160226-RG-decision-tree-for-Wizard-CC-BY-26- February-2016.pdf), we found that none of the most popular checklists are appropriate for our study design.

Acknowledgements: The authors would like to thank Erinma Elibe, MPH and Kristin Zerfas for their contributions toward creating and distributing the online survey, respectively. We would also like to acknowledge members of the Johns Hopkins Neuro-Oncology Surgical Outcomes Lab for their valuable input that has helped inform this project.

Funding: The authors acknowledge assistance for clinical data coordination and retrieval from the Core for Clinical Research Data Acquisition, supported in part by the Johns Hopkins Institute for Clinical and Translational Research (UL1TR001079). The authors received no remuneration for the authorship and/or publication of this article.

Competing Interests: The authors have no relevant financial or non-financial interests to disclose.

Authorship: MR: Methodology, Formal Analysis, Writing-Original Draft, Review and Editing. AMK: Conceptualization, Methodology. OW: Formal Analysis, Writing-Original Draft, Review and Editing. DD: Formal Analysis, Writing-Original Draft, Review and Editing. JPS: Data Acquisition, Writing-Review and Editing. JHS: Data Acquisition, Writing-Review and Editing. DM: Conceptualization, Supervision, WritingReview and Editing.

Data Availability: The datasets generated during and/or analysed during the current study are available from the corresponding authors on reasonable request.

Ethics Approval: This study was reviewed and approved by the Johns Hopkins institutional review board (IRB00196609).

Consent to Participate and Publish: This study was exempt from obtaining patient consent from patients whose radiographic data was used to generate the survey, as all data was de-identified as required by Health Insurance Portability and Accountability Act (HIPAA) regulations and institutional review board protocol (IRB00196609). Informed consent was obtained from all participants who completed the crowdsourcing survey.

\section{References}

1. Omuro A, DeAngelis L (2013) Glioblastoma and Other Malignant Gliomas: A Clinical Review. JAMA 310(17):1842-1850. doi:10.1001/jama.2013.280319

2. Ostrom QT, Gittleman H, Liao P et al (2014) CBTRUS statistical report: Primary brain and central nervous system tumors diagnosed in the United States in 2007-2011. Neuro Oncol 16:iv1-iv63. doi:10.1093/neuonc/nou223 
3. Louis DN, Perry A, Reifenberger G et al (2016) The 2016 World Health Organization Classification of Tumors of the Central Nervous System: a summary. Acta Neuropathol 131(6):803-820. doi:10.1007/s00401-016-1545-1

4. Stupp R, Mason W, Bent M, van den et al (2005) Radiotherapy plus concomitant and adjuvant temozolomide for glioblastoma. N Engl J Med 352(10):987-996. doi:10.4137/cmo.s390

5. Price SJ, Gillard JH (2011) Imaging biomarkers of brain tumour margin and tumour invasion. $\mathrm{Br} \mathrm{J}$ Radiol. ;84(SPEC. ISSUE 2):159-167. doi:10.1259/bjr/26838774

6. Simpson JR, Horton J, Scott C et al (1993) Influence of location and extent of surgical resection on survival of patients with glioblastoma multiforme: Results of three consecutive radiation therapy oncology group (RTOG) clinical trials. Int J Radiat Oncol Biol Phys 26(2):239-244. doi:10.1016/0360-3016(93)90203-8

7. Jeremic B, Grujicic D, Antunovic V, Djuric L, Stojanovic M, Shibamoto Y (1994) Influence of extent of surgery and tumor location on treatment outcome of patients with glioblastoma multiforme treated with combined modality approach. J Neurooncol 21(2):177-185. doi:10.1007/BF01052902

8. Ushio Y, Kochi M, Hamada JI, Kai Y, Nakamura H (2005) Effect of surgical removal on survival and quality of life in patients with supratentorial glioblastoma. Neurol Med Chir (Tokyo) 45(9):454-460. doi:10.2176/nmc.45.454

9. Stummer W, Reulen H, Meinel T (2008) Extent of Resection and survival in glioblastoma multiforme: indentification of and adjustment for bias. Neurosurgery 62(3):564-576. doi:10.1227/01.NEU.0000297118.47076.5E

10. Davis ME, Glioblastoma (2016) Overview of Disease and Treatment. Clin J Oncol Nurs 20(5):S2-S8

11. De Leeuw CN, Vogelbaum MA (2019) Supratotal resection in glioma: A systematic review. Neuro Oncol 21(2):179-188. doi:10.1093/neuonc/noy166

12. Duffau $\mathrm{H}$ (2012) Awake surgery for incidental WHO grade Il gliomas involving eloquent areas. Acta Neurochir (Wien) 154(4):575-584. doi:10.1007/s00701-011-1216-x

13. Incekara F, Koene S, Vincent AJPE, van den Bent MJ, Smits M (2019) Association Between Supratotal Glioblastoma Resection and Patient Survival: A Systematic Review and Meta-Analysis. World Neurosurg 127:617-624e2. doi:10.1016/j.wneu.2019.04.092

14. Jackson C, Choi J, Khalafallah AM et al (2020) A systematic review and meta-analysis of supratotal versus gross total resection for glioblastoma. J Neurooncol 148(3):419-431. doi:10.1007/s11060020-03556-y

15. Khalafallah AM, Huq S, Jimenez AE, Serra R, Bettegowda C, Mukherjee D (2021) "zooming in" on Glioblastoma: Understanding Tumor Heterogeneity and its Clinical Implications in the Era of SingleCell Ribonucleic Acid Sequencing. Neurosurgery 88(3):477-486. doi:10.1093/neuros/nyaa305

16. Khalafallah AM, Rakovec M, Bettegowda C et al (2021) A Crowdsourced Consensus on Supratotal Resection Versus Gross Total Resection for Anatomically Distinct Primary Glioblastoma. Neurosurgery 0(0):1-8. doi:10.1093/neuros/nyab257 
17. Kitabayashi T, Nakada M, Furuta T et al (2014) NC-09: The Impact of Supratotal Resection for Glioblastoma. Neuro Oncol 16:v134-v137

18. Molinaro AM, Hervey-Jumper S, Morshed RA et al (2020) Association of Maximal Extent of Resection of Contrast-Enhanced and Non-Contrast-Enhanced Tumor with Survival Within Molecular Subgroups of Patients with Newly Diagnosed Glioblastoma. JAMA Oncol 6(4):495-503. doi:10.1001/jamaoncol.2019.6143

19. Li YM, Suki D, Hess K, Sawaya R (2016) The influence of maximum safe resection of glioblastoma on survival in 1229 patients: Can we do better than gross-total resection? J Neurosurg 124(4):977988. doi:10.3171/2015.5.JNS142087

20. Mampre D, Ehresman J, Pinilla-Monsalve $\mathrm{G}$ et al (2018) Extending the resection beyond the contrastenhancement for glioblastoma: feasibility, efficacy, and outcomes. Br J Neurosurg 32(5):528-535. doi:10.1080/02688697.2018.1498450

21. De Bonis $P$, Anile $C$, Pompucci A et al (2013) The influence of surgery on recurrence pattern of glioblastoma. Clin Neurol Neurosurg 115(1):37-43. doi:10.1016/j.clineuro.2012.04.005

22. Glenn CA, Baker CM, Conner AK et al (2018) An Examination of the Role of Supramaximal Resection of Temporal Lobe Glioblastoma Multiforme. World Neurosurg 114:e747-e755. doi:10.1016/j.wneu.2018.03.072

23. Esquenazi Y, Friedman E, Liu Z, Zhu JJ, Hsu S, Tandon N (2017) The Survival Advantage of "supratotal" Resection of Glioblastoma Using Selective Cortical Mapping and the Subpial Technique. Neurosurgery 81(2):275-288. doi:10.1093/neuros/nyw174

24. Hamada S, Abou-Zeid A (2016) Anatomical resection in glioblastoma: extent of resection and its impact on duration of survival. Egypt J Neurol Psychiatry Neurosurg 53(3):135-145. doi:10.4103/1110-1083.192655

25. Lu M, Fu Z, hao, He X (2020) T2 Fluid-Attenuated Inversion Recovery Resection for Glioblastoma Involving Eloquent Brain Areas Facilitated Through Awake Craniotomy and Clinical Outcome. World Neurosurg 135:e738-e747. doi:10.1016/j.wneu.2019.12.130

26. Pessina F, Navarria P, Cozzi L et al (2017) Maximize surgical resection beyond contrast-enhancing boundaries in newly diagnosed glioblastoma multiforme: is it useful and safe? A single institution retrospective experience. J Neurooncol 135(1):129-139. doi:10.1007/s11060-017-2559-9

27. Aldave G, Tejada S, Pay E et al (2013) Prognostic value of residual fluorescent tissue in glioblastoma patients after gross total resection in 5-aminolevulinic acid-guided surgery. Neurosurgery 72(6):915920. doi:10.1227/NEU.0b013e31828c3974

28. Spetzler RF, Martin NA (1986) A proposed grading system for arteriovenous malformations. $J$ Neurosurg. 2008;108(1):186-193. doi:10.3171/JNS/2008/108/01/0186

29. Friedlein K, Bozhkov Y, Hore $\mathrm{N}$ et al (2015) A new functional classification system (FGA/B) with prognostic value for glioma patients. Sci Rep 5:1-11. doi:10.1038/srep12373

30. Kahn E, Lane M, Sagher O, Eloquent (2017) History of a word's adoption into the neurosurgical lexicon. J Neurosurg 127(6):1461-1466. doi:10.3171/2017.3.JNS17659 
31. Fried I (1993) The Myth of Eloquent Cortex, or What is Non-Eloquent Cortex? J Neurosurg 78:10091010. doi:10.3171/2014.12.JNS142826

32. Ghogawala Z, Schwartz JS, Benzel EC et al (2016) Increased patient enrollment to a randomized surgical trial through equipoise polling of an expert surgeon panel. Ann Surg 264(1):81-86. doi:10.1097/SLA.0000000000001483

33. Khalafallah AM, Rakovec M, Bettegowda C et al (2021) A Crowdsourced Consensus on Supratotal Resection Versus Gross Total Resection for Anatomically Distinct Primary Glioblastoma. Neurosurgery 89(4):712-719. doi:10.1093/neuros/nyab257

34. Sonabend AM, Zacharia BE, Cloney MB et al (2017) Defining glioblastoma resectability through the wisdom of the crowd: A proof-of-principle study. Neurosurgery 80(4):590-601. doi:10.1227/NEU.0000000000001374

35. Choucair A, Levin V, Gutin P et al (1986) Development of multiple lesions during radiation and chemotherapy in patients With Gliomas. J Neurosurg 65:654-658

36. Hou LC, Veeravagu A, Hsu AR, Tse VCK (2006) Recurrent glioblastoma multiforme: a review of natural history and management options. Neurosurg Focus 20(4). doi:10.3171/foc.2006.20.4.2

37. Gaspar LE, Fisher BJ, Macdonald DR et al (1992) Supratentorial malignant glioma: Patterns of recurrence and implications for external beam local treatment. Int J Radiat Oncol Biol Phys 24(1):55-57. doi:10.1016/0360-3016(92)91021-E

38. Burger PC, Dubois PJ, Schold SC et al (1983) Computerized tomographic and pathologic studies of the untreated, quiescent, and recurrent glioblastoma multiforme. J Neurosurg 58(2):159-169. doi:10.3171/jns.1983.58.2.0159

39. Petrecca K, Guiot MC, Panet-Raymond V, Souhami L (2013) Failure pattern following complete resection plus radiotherapy and temozolomide is at the resection margin in patients with glioblastoma. J Neurooncol 111(1):19-23. doi:10.1007/s11060-012-0983-4

40. Darmanis S, Sloan SA, Croote D et al (2017) Single-Cell RNA-Seq Analysis of Infiltrating Neoplastic Cells at the Migrating Front of Human Glioblastoma. Cell Rep 21(5):1399-1410. doi:10.1016/j.celrep.2017.10.030

41. Schneider M, Potthoff AL, Keil VC et al (2019) Surgery for temporal glioblastoma: lobectomy outranks oncosurgical-based gross-total resection. J Neurooncol 145(1):143-150. doi:10.1007/s11060-019-03281-1

42. Shah AH, Mahavadi A, Di L et al (2020) Survival benefit of lobectomy for glioblastoma: moving towards radical supramaximal resection. J Neurooncol 148(3):501-508. doi:10.1007/s11060-02003541-5

43. Roh TH, Kang SG, Moon JH et al (2020) Survival benefit of lobectomy over gross-total resection without lobectomy in cases of glioblastoma in the noneloquent area: A retrospective study. $J$ Neurosurg 132(3):895-901. doi:10.3171/2018.12.JNS182558

44. McGirt MJ, Mukherjee D, Chaichana KL, Than KD, Weingart JD, Quinones-Hinojosa A (2009) Association of surgically acquired motor and language deficits on overall survival after resection of 
glioblastoma multiforme. Neurosurgery 65(3):463-469. doi:10.1227/01.NEU.0000349763.42238.E9

45. Rahman M, Abbatematteo J, De Leo EK et al (2017) The effects of new or worsened postoperative neurological deficits on survival of patients with glioblastoma. J Neurosurg 127(1):123-131. doi:10.3171/2016.7.JNS16396

46. Bu LH, Zhang J, Lu JF, Wu JS (2021) Glioma surgery with awake language mapping versus generalized anesthesia: a systematic review. Neurosurg Rev 44(4):1997-2011. doi:10.1007/s10143020-01418-9

47. Sanai N, Berger M (2012) Recent Surgical Management of Gliomas.. In: In: Yamanaka R (ed) Glioma. Advances in Experimental Medicine and Biology. Springer, New York, NY, pp 12-25

48. Hervey-Jumper SL, Berger MS (2019) Insular glioma surgery: An evolution of thought and practice. J Neurosurg 130(1):9-16. doi:10.3171/2018.10.JNS181519

49. Sanai N, Polley MY, Berger MS (2010) Insular glioma resection: Assessment of patient morbidity, survival, and tumor progression - Clinical article. J Neurosurg 112(1):1-9.

doi:10.3171/2009.6.JNS0952

50. Alimohamadi M, Shirani M, Shariat Moharari R et al (2016) Application of Awake Craniotomy and Intraoperative Brain Mapping for Surgical Resection of Insular Gliomas of the Dominant Hemisphere. World Neurosurg 92:151-158. doi:10.1016/j.wneu.2016.04.079

\section{Figures}

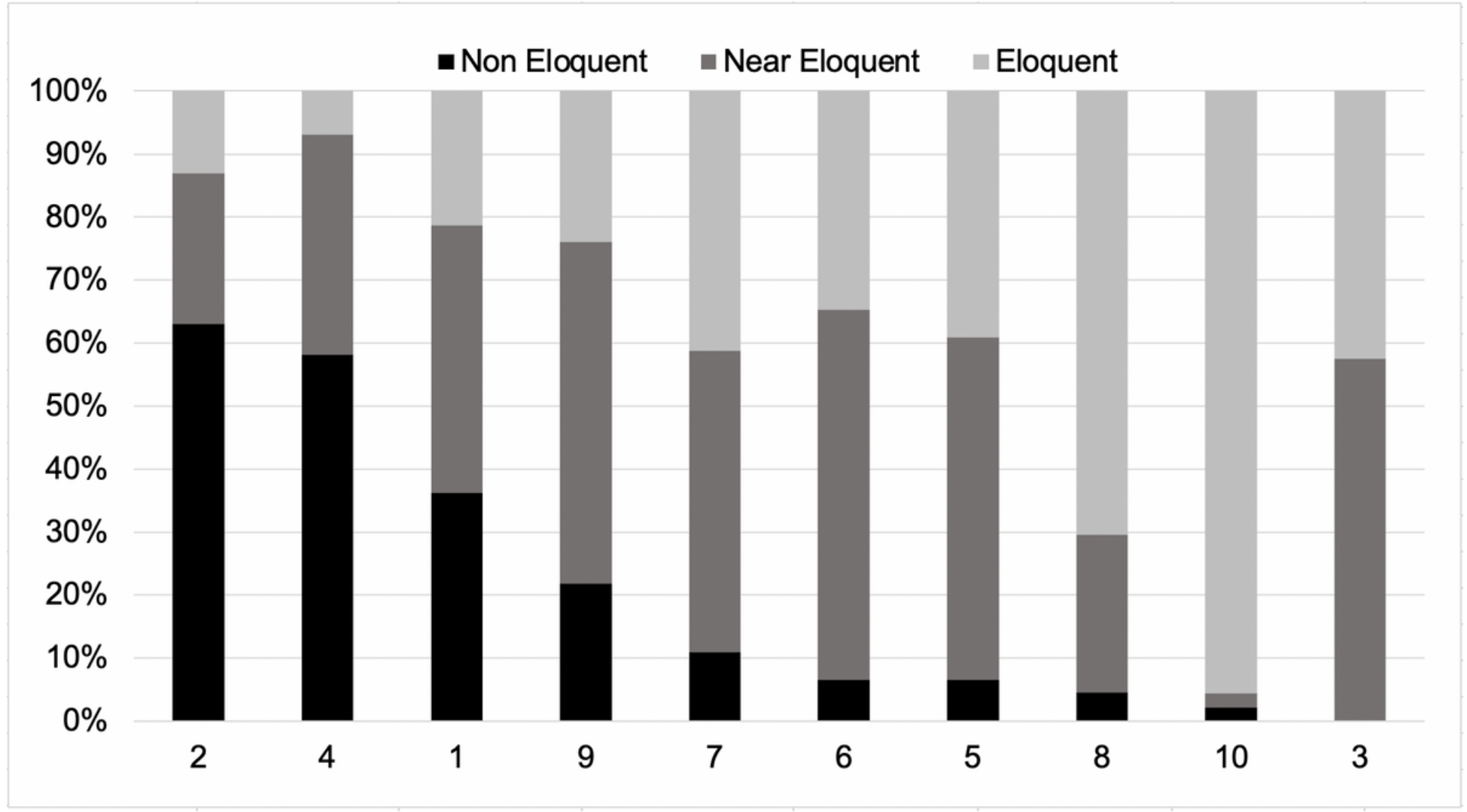


The percentage of neurosurgical oncologists who rated each GBM's location as non-eloquent, near eloquent, or eloquent. R: Right; L: Left; Ant: Anterior; Post: Posterior

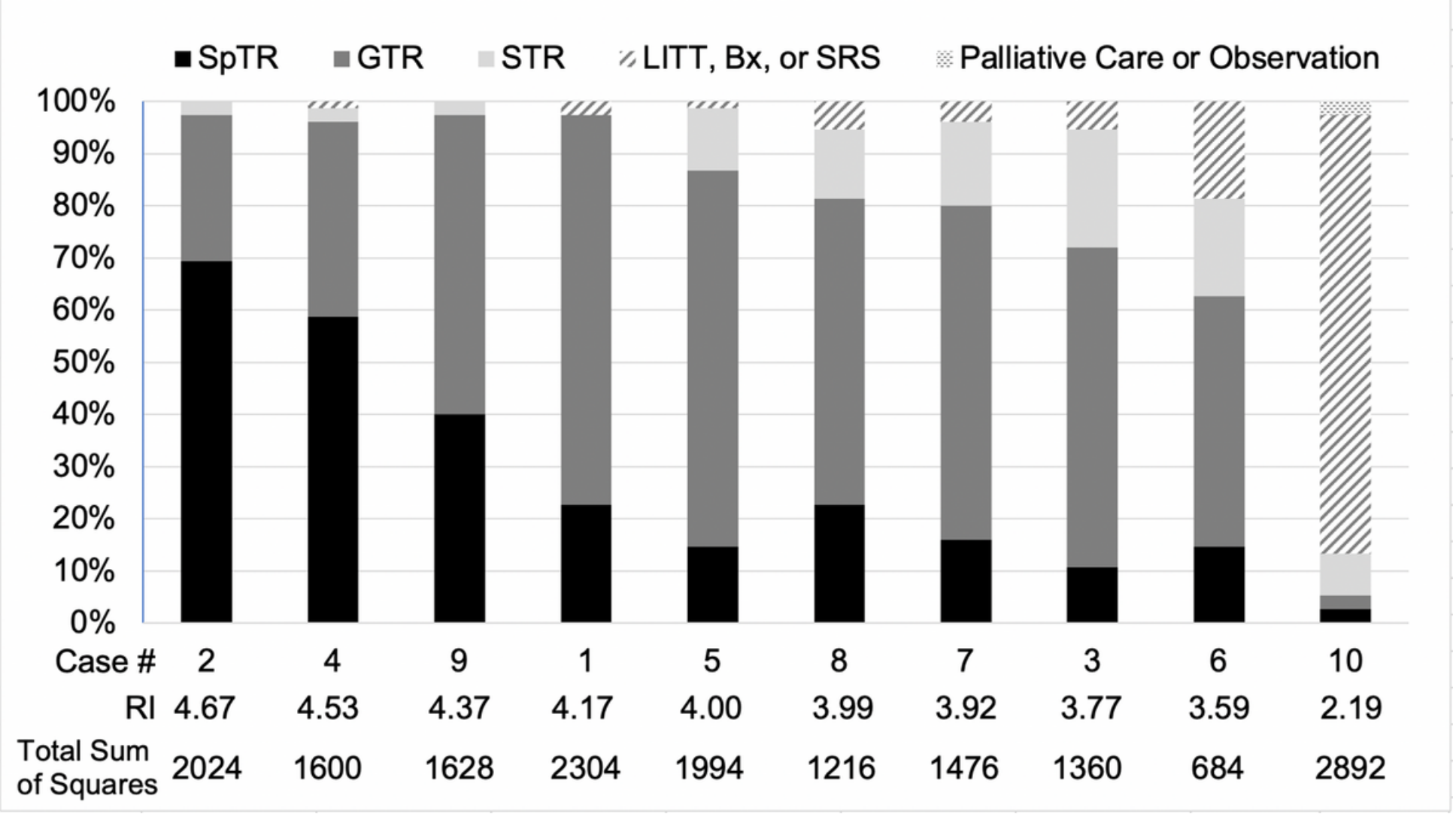

Figure 2

The percentage of neurosurgical oncologists who selected palliative care or observation; laser interstitial thermal therapy (LITT), stereotactic biopsy, excisional biopsy (Bx), or stereotactic radiosurgery (SRS); subtotal resection (STR); gross total resection (GTR); or supratotal resection (SpTR) for each case. The resectability index (RI) and total sum of squares for each case are listed below. A higher RI value represents a GBM that is considered on average to be amenable to more aggressive treatment, such as SpTR; a higher total sum of squares indicates greater agreement between surgeons on proposed treatment plan. Differences between cases' RIs are statistically significant as determined by ANOVA ( $p<$ 0.0005). R: Right; L: Left; Ant: Anterior; Post: Posterior

\section{Supplementary Files}

This is a list of supplementary files associated with this preprint. Click to download.

- TumorSectionCrowdsourcingSupplement11.17.21.docx

- eAppendixFullSurvey.pdf 